\title{
Multilocus Variable-Number Tandem-Repeat Analysis, Pulsed-Field Gel Electrophoresis, and Antimicrobial Susceptibility Patterns in Discrimination of Sporadic and Outbreak-Related Strains of Yersinia enterocolitica
}

\author{
Leila M Sihvonen $^{1 *}$, Susanna Toivonen ${ }^{3}$, Kaisa Haukka', Markku Kuusi², Mikael Skurnik ${ }^{3,4}$, Anja Siitonen ${ }^{1}$
}

\begin{abstract}
Background: We assessed the potential of multilocus variable-number tandem-repeat analysis (MLVA), pulsed-field gel electrophoresis (PFGE), and antimicrobial susceptibility testing for discriminating 104 sporadic and outbreakrelated Yersinia enterocolitica (YE) bio/serotype 3-4/O:3 and 2/0:9 isolates. MLVA using six VNTR markers was performed in two separate multiplex PCRs, and the fluorescently labeled PCR products were accurately sized on an automated DNA sequencer.

Results: MLVA discriminated 82 sporadic YE 3-4/O:3 and 2/O:9 strains into 77 types, whereas PFGE with the restriction enzyme Notl discriminated the strains into 23 different PFGE pulsotypes. The discriminatory index for a sporadic strain was 0.862 for PFGE and 0.999 for MLVA. MLVA confirmed that a foodborne outbreak in the city of Kotka, Finland in 2003 had been caused by a multiresistant YE 4/O:3 strain that was distinctly different from those of epidemiologically unrelated strains with an identical PFGE pulsotype. The multiresistance of $Y$. enterocolitica strains (19\% of the sporadic strains) correlated significantly $(p=0.002)$ with travel abroad. All of the multiresistant Y. enterocolitica strains belonged to four PFGE pulsotypes that did not contain any susceptible strains. Resistance to nalidixic acid was related to changes in codons 83 or 87 that stemmed from mutations in the gyrA gene. The conjugation experiments demonstrated that resistance to CHL, STR, and SUL was carried by a conjugative plasmid.

Conclusions: MLVA using six loci had better discriminatory power than PFGE with the Notl enzyme. MLVA was also a less labor-intensive method than PFGE and the results were easier to analyze. The conjugation experiments demonstrated that a resistance plasmid can easily be transferred between $Y$. enterocolitica strains. Antimicrobial multiresistance of $Y$. enterocolitica strains was significantly associated with travel abroad.
\end{abstract}

\section{Background}

Yersinia enterocolitica (YE) is an enteropathogenic bacterium transmitted via food or water and may cause sporadic infections as well as foodborne outbreaks of yersiniosis [1-5]. The symptoms of yersiniosis range from mild diarrhea to severe clinical manifestations and post-infectious complications such as reactive arthritis,

\footnotetext{
* Correspondence: leila.sihvonen@thl.fi

'Department of Infectious Disease Surveillance and Control, Bacteriology Unit, National Institute for Health and Welfare (THL), Helsinki, Fl-00271, Finland

Full list of author information is available at the end of the article
}

myocarditis, glomerulonephritis, and erythema nodosum [6]. Y. enterocolitica can be divided into six biotypes, of which biotypes $1 \mathrm{~B}$ and $2-5$ are known to be pathogenic to humans.

At present, pulsed-field gel electrophoresis (PFGE) is commonly used to discriminate between $Y$. enterocolitica strains. However, there are no standard PFGE procedures or databases similar to those, e.g., for Escherichia coli O157:H7, Salmonella, and Shigella standardized by PulseNet [7]. Most of the restriction enzymes used in PFGE for $Y$. enterocolitica produce patterns with a high number of bands that are not ideal for

\section{Ciomed Central}


analysis. Furthermore, the global homogeneity of the pulsotypes among $Y$. enterocolitica 4/O:3 is high and different pulsotypes often display only minor differences [8-11]. However, the discriminatory power of PFGE has been improved by using more than one restriction enzyme [12].

Most bacterial genomes contain repeats of DNA sequences called "variable-number tandem repeats" (VNTR). These VNTR regions can be applied in the PCR-based subtyping of strains by multilocus variablenumber tandem-repeat analysis (MLVA). MLVA is increasingly used for typing, surveillance and epidemiological investigations of pathogenic bacteria [13]. A study investigating the development of an MLVA subtyping method to be used for $Y$. enterocolitica 4/O:3, based on six loci, was reported recently [14].

Although yersiniosis is seldom treated with antimicrobials, medication may be required, for example in the case of immuno-compromised patients. $Y$. enterocolitica is a known $ß$-lactamase producer and thus is resistant to $B$-lactam antibiotics such as ampicillin, carbenicillin, penicillin, and first-generation cephalosporins [15-20]. In recent studies done in Switzerland, the USA, Germany, and Austria, $Y$. enterocolitica strains have shown high susceptibility to antimicrobials other than ß-lactams [21-24]. However, multiresistant Y. enterocolitica strains have also been reported, e.g., from Spain and Brazil $[16,25,26]$. The antimicrobial resistance of $Y$. enterocolitica has not been monitored regularly in Finland although the surveillance of antimicrobial resistance would be useful for epidemiological studies. Over 20 years ago, 186 Finnish $Y$. enterocolitica strains were studied and found to be resistant only to ampicillin and susceptible to ceftriaxone, tetracycline, sulphatrimethoprim, and ciprofloxacin [27].

The aim of the present study was to determine how MLVA using fluorescently labeled primers and fragment analysis compares to PFGE in its discriminatory power with regard to the sporadic and outbreak-related strains of YE bio/serotypes 4/O:3. We included traditional antimicrobial susceptibility testing in our study to see whether it provides additional information for the genotypic analysis concerning, e.g., the geographical source of infection. We therefore used MLVA and PFGE to type 104 sporadic and outbreak-associated $Y$. enterocolitica strains and determined the sensitivity of the strains to 12 antimicrobial agents. In addition, we studied the genetic basis of the antimicrobial resistance that was detected.

\section{Results}

\section{Sporadic strains}

The strains isolated in $2006(\mathrm{n}=82)$ were discriminated into 77 types by MLVA (Figure 1) and into 23 pulsotypes by PFGE (Figure 2). There were two YE 4/O:3 strains with identical MLVA types in only five cases. In two of these cases, the identical strains had been isolated from one patient 7 days apart and from another patient 19 days apart. The discriminatory index for sporadic strains was 0.862 for PFGE and 0.999 for MLVA.

The six loci used in MLVA V2A exhibited the highest discriminatory power $(\mathrm{DI}=92 \%$ ), resolving 17 different alleles. The least variation was observed for locus V9 $(\mathrm{DI}=62 \%)$, which yielded only six different alleles, i.e., 2-7 repeats of a repetitive sequence $12 \mathrm{bp}$ in length. The discriminatory indexes of loci V4, V5, V6, and V7 were $71,89,91$, and $90 \%$, respectively. The fragment sizes defined by the capillary electrophoresis of the six VNTR loci and the number of repeats confirmed by DNA sequencing are shown in Table 1.

\section{Suspected outbreak strains}

The suspected outbreak strains $(\mathrm{n}=22)$ from December 2003 to January 2004 represented four PFGE pulsotypes, which were further typed into 11 MLVA types. Twelve of the strains were identical in MLVA type. Eleven of these strains with identical MLVA types were isolated from the patients with an epidemiological connection to the disease outbreak. The 12 strains with identical MLVA type represented 2 slightly different (only one band difference) PFGE pulsotypes (Figure 2) and were multiresistant to antimicrobials (Figure 1). Among these strains, eleven were resistant to AMP, CHL, STR SUL, and TET; one strain was susceptible to TET. The suspected outbreak strains with different MLVA types did not have a proved connection to the city of Kotka, Finland. Nine of these strains were susceptible to all the tested antimicrobials except AMP and eight of them shared the same PFGE type. One of the strains (IH250258) had an antimicrobial resistance profile and a PFGE pulsotype identical to those of the outbreak strains. However, the different MLVA type and the lack of epidemiological connection distinguished this particular case from the outbreak-associated cases (Figure 2). Suspected YE 4/O:3 outbreak strains isolated in 2006 from six 1-year-old children displayed the same PFGE pulsotype (5NotI_ye a). However, the MLVA discriminated all six strains.

\section{Association between the antimicrobial resistance and travel}

All the $Y$. enterocolitica strains studied here were resistant to ampicillin. Fifteen (19\%) of 80 sporadic strains isolated in 2006 from 80 patients were resistant to four or five of the antimicrobials tested (Table 2). The multiresistant strains belonged to certain PFGE pulsotypes (1NotI_ye, 3NotI_ye, 7NotI_ye, 15NotI_ye) that did not contain any susceptible strains. The travel history of 70 of the 80 patients was known. Of these patients, 46\% (32/70) had traveled abroad before the onset of symptoms. Travel abroad was significantly $(p=0.002)$ associated with the 


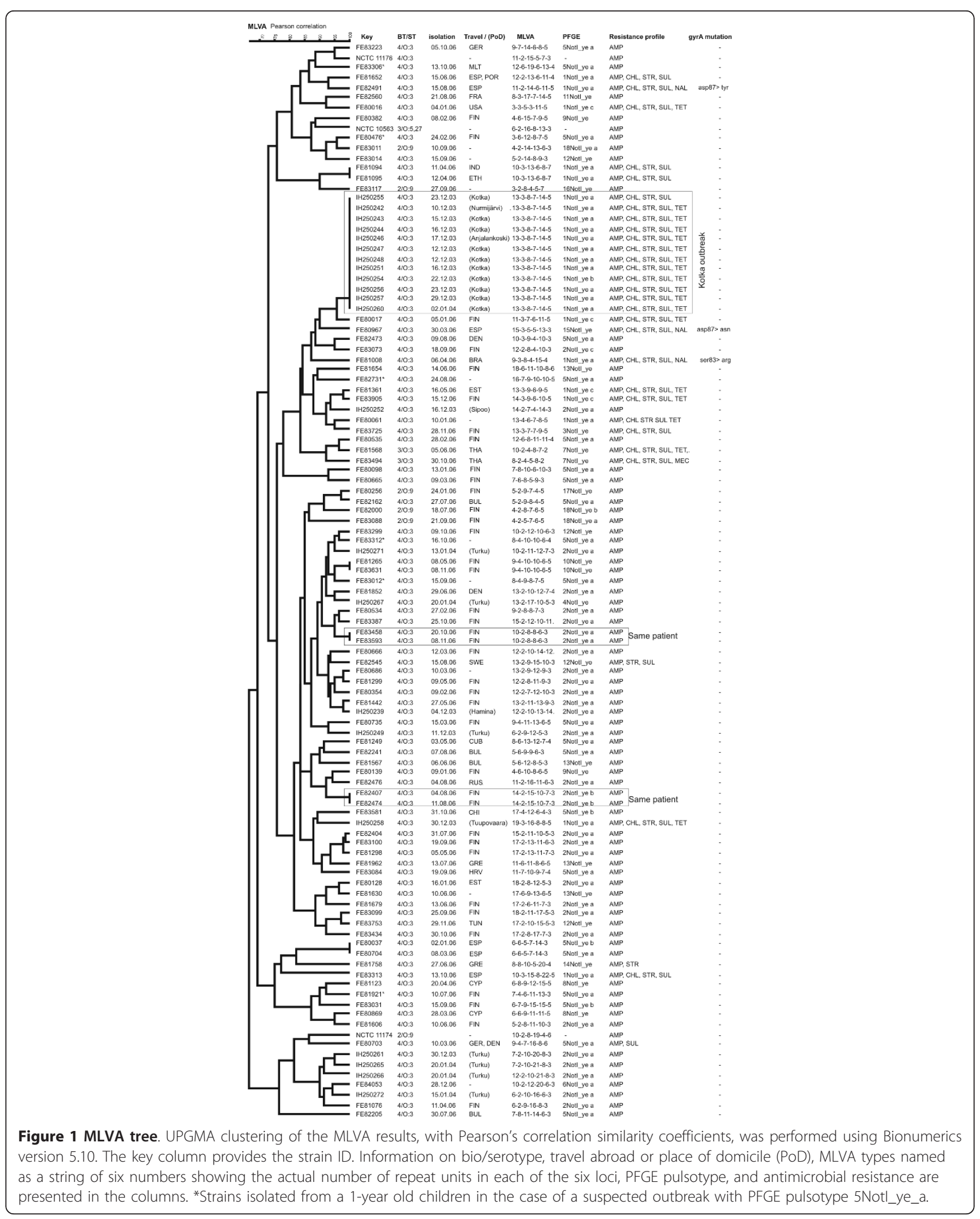




\section{Different PFGE types (n=24)}

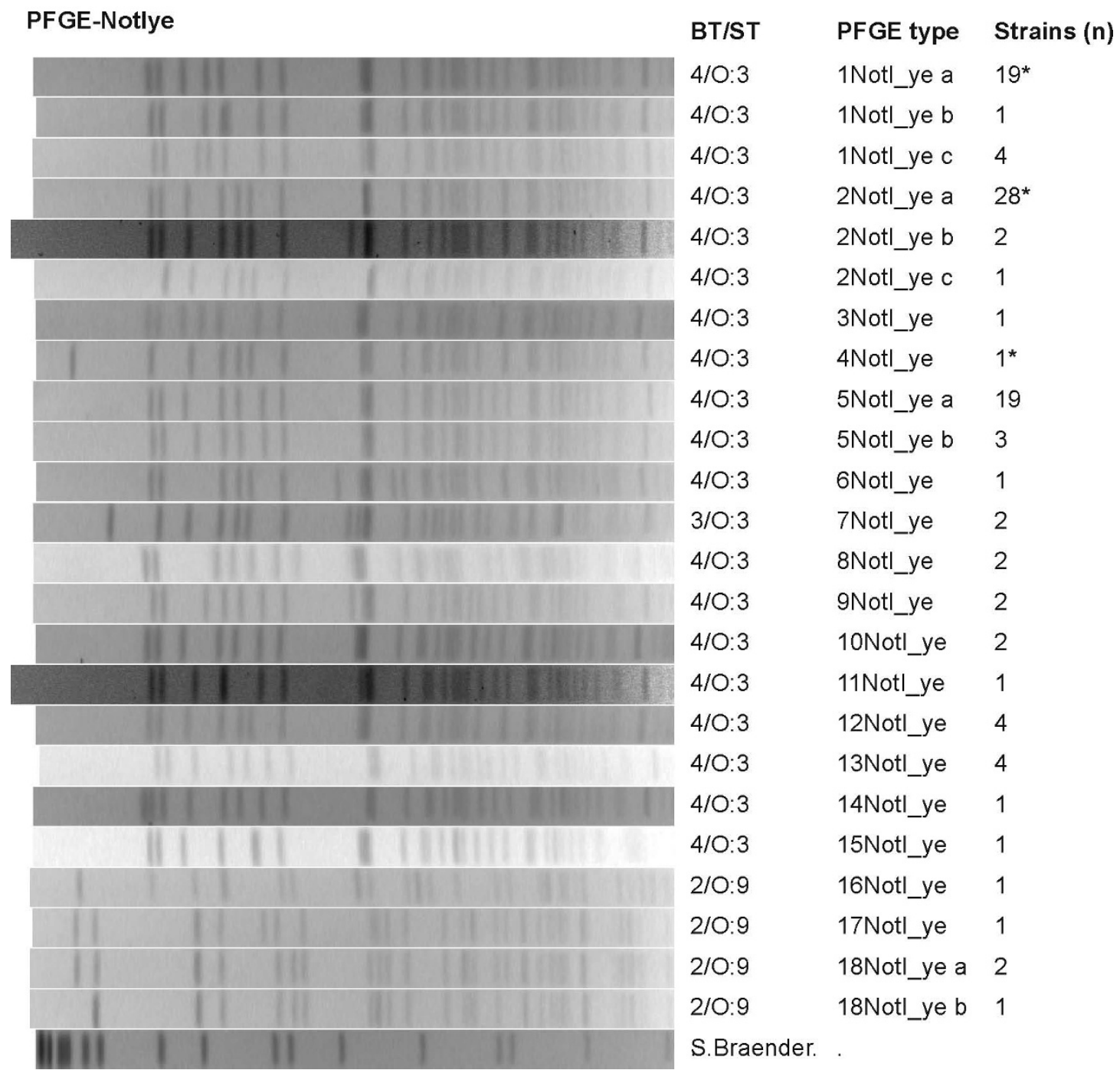

Figure 2 PFGE types of the studied strains. All 24 representative PFGE types of 104 strains in the present study. * The strain number includes the outbreak types.

antimicrobial multiresistance of $Y$. enterocolitica: 34\% (11/ $32)$ of the patients with and $5 \%(2 / 38)$ of the patients without a trip abroad had a multiresistant $Y$. enterocolitica strain. Three strains resistant to nalidixic acid had decreased susceptibility $(0.25,0.25$, or $0.5 \mathrm{mg} / \mathrm{L})$ to ciprofloxacin in MIC determination. Sequencing of these three nalidixic acid resistant strains revealed amino acid changes due to the point mutations in the gyrA gene; i.e., Ser83 to Arg or Asp87 to Asn or Asp87 to Tyr.

\section{Conjugation of resistance plasmid}

In the conjugation experiment, a sporadic YE 4/O:3 strain FE81008 (resistant to AMP, CHL, STR, SUL, and
NAL) was able to transfer the CHL, STR, and SUL resistances to strain $\mathrm{YeO} 3-\mathrm{U}$ by conjugation. The conjugation frequency was $10^{-5}-10^{-6}$. This indicated that the genes encoding resistance to CHL, STR, and SUL were carried on a conjugative plasmid. Indeed, plasmid isolation demonstrated that the recipient strain had received a large 30-40 kb plasmid.

\section{Discussion}

In our study, MLVA typing using fluorescently labeled primers and fragment analysis was shown to be a highresolution discriminatory method for epidemiological investigations of $Y$. enterocolitica. In the present study, 
Table 1 Diversity of VNTR alleles

\begin{tabular}{|c|c|c|c|c|c|c|c|c|c|c|c|c|}
\hline $\begin{array}{l}\text { Number of the } \\
\text { repeats }\end{array}$ & $\begin{array}{l}\text { V2A TCTCAC } \\
\text { (bp) }\end{array}$ & $\mathrm{nt}$ & $\begin{array}{l}\text { V4 CGGCAAC } \\
\text { (bp) }\end{array}$ & $\mathrm{n}$ & $\begin{array}{c}\text { V5 GGTGCA } \\
\text { (bp) }\end{array}$ & $\mathrm{n}$ & $\begin{array}{l}\text { V6 GACTCA } \\
\text { (bp) }\end{array}$ & $\mathrm{n}$ & $\begin{array}{l}\text { V7 GTGCTG } \\
\text { (bp) }\end{array}$ & $\mathrm{n}$ & $\begin{array}{c}\text { V9 } \\
\text { ATGTCGGTAGAA } \\
\text { (bp) }\end{array}$ & $\bar{n}$ \\
\hline 2 & - & & $119^{*}$ & 49 & & & - & & - & & 108 & 2 \\
\hline 3 & $246^{*}$ & 2 & $126^{*}$ & 26 & & & 182 & 1 & - & & $120^{*}$ & 52 \\
\hline 4 & 252 & 5 & $133^{*}$ & 9 & 199 & 2 & $188^{*}$ & 5 & $195^{*}$ & 4 & 132 & 8 \\
\hline 5 & 258 & 6 & 140 & 0 & 205 & 4 & 194 & 5 & $201^{*}$ & 8 & $144^{*}$ & 40 \\
\hline 6 & 264 & 10 & 147 & 15 & 211 & 3 & $200^{*}$ & 11 & 207 & 19 & 156 & 2 \\
\hline 7 & 270 & 6 & $154^{*}$ & 4 & 217 & 6 & $206^{*}$ & 21 & 213 & 13 & $168^{*}$ & 3 \\
\hline 8 & 276 & 6 & 161 & 4 & $223^{*}$ & 25 & $212^{*}$ & 13 & 219 & 12 & - & \\
\hline 9 & $282^{*}$ & 7 & - & & 229 & 17 & 218 & 2 & 225 & 9 & - & \\
\hline 10 & 288 & 10 & - & & 235 & 15 & 224 & 12 & 231 & 9 & - & \\
\hline 11 & 294 & 6 & - & & 241 & 8 & 230 & 9 & 237 & 7 & - & \\
\hline 12 & 300 & 10 & - & & 247 & 6 & 236 & 10 & 243 & 1 & - & \\
\hline 13 & 306 & 20 & - & & 253 & 6 & 242 & 4 & 249 & 4 & - & \\
\hline 14 & 312 & 4 & - & & 259 & 5 & 248 & 2 & $255^{*}$ & 16 & - & \\
\hline 15 & 318 & 3 & - & & $265^{*}$ & 5 & 254 & 3 & 261 & 3 & - & \\
\hline 16 & 324 & 1 & - & & 271 & 3 & 260 & 3 & 267 & - & - & \\
\hline 17 & $330^{*}$ & 7 & - & & 277 & 1 & 266 & 2 & 273 & - & - & \\
\hline 18 & 336 & 3 & - & & 283 & - & 272 & - & 279 & - & - & \\
\hline 19 & 342 & 1 & - & & 290 & 1 & 278 & - & 285 & - & - & \\
\hline 20 & - & & - & & - & & 284 & 2 & 291 & 1 & - & \\
\hline 21 & - & & - & & - & & 300 & 2 & 297 & - & - & \\
\hline 22 & - & & - & & - & & & & $303^{*}$ & 1 & - & \\
\hline
\end{tabular}

Fragment sizes (bp) defined by capillary electrophoresis of VNTR alleles with different number of repeats and their diversity in 107 studied $Y$. enterocolitica strains.

* Alleles were sequenced to confirm the number of repeats.

$+\mathrm{n}=$ number of strains.

the discriminatory power of MLVA was $99.9 \%$ while that of NotI PFGE was $87.9 \%$. Our results were in agreement to those obtained by Gierczyński and colleagues [14] who demonstrated that the used MLVA markers are highly discriminatory and added the evidence that this method can successfully be applied for the outbreak strains of $Y$. enterocolitica ssp. palearctica biotypes 2 and 4. In the present study, only the VNTR loci V2A,
V4 and V5 were found in six BT 1A strains tested with the MLVA method (data not shown). Another MLVA method designed using $Y$. enterocolitica ssp. enterocolitica strain 8081 whole genome and with four loci was introduced recently [28]. The method showed potential for the epidemiological investigation for YE biotype 1A strains with DI of $87 \%$ and worked also for six tested BT 2 and BT4 strains [28]. The discriminatory power of

Table 2 Antimicrobial resistance and travelling

\begin{tabular}{|c|c|c|c|c|}
\hline Resistance profile & $\begin{array}{l}\text { Suspected outbreak YE 4/0:3 } \\
\qquad(n=22)\end{array}$ & $\begin{array}{c}\text { Sporadic YE } 4 / 0: 3 \\
(n=75)\end{array}$ & $\begin{array}{l}\text { Sporadic YE } 3 / 0: 3 \\
(n=2)\end{array}$ & $\begin{array}{l}\text { Sporadic YE 2/O:9 } \\
(n=5)\end{array}$ \\
\hline AMP CHL STR SUL NAL & - & $3(100 \%)^{*}$ & - & - \\
\hline AMP CHL STR SUL TET & $12(0 \%)$ & $5(40 \%)$ & - & - \\
\hline $\begin{array}{l}\text { AMP CHL STR SUL TET } \\
\text { MEC }\end{array}$ & - & - & $1(100 \%)$ & - \\
\hline AMP CHL STR SUL & $1(0 \%)$ & $5(80 \%)$ & - & - \\
\hline AMP CHL STR SUL MEC & - & - & $1(100 \%)$ & - \\
\hline AMP SUL & - & $1(100 \%)$ & - & - \\
\hline AMP STR & - & 1 & - & - \\
\hline AMP MEC & - & - & - & - \\
\hline AMP & $9(0 \%)$ & $61(28 \%)$ & - & $5(0 \%)$ \\
\hline
\end{tabular}

Antimicrobial resistance profiles of the sporadic $Y$. enterocolitica strains isolated in Finland in 2006 and suspected outbreak strains from $2003-2004$ and related travel information.

* The percentage of the patients who had reported having traveled abroad before getting ill is in the parenthesis. 
PFGE can be improved by using more than one restriction enzyme. For instance, the discriminatory index of $74 \%$ achieved with Not I PFGE was increased to $93 \%$ by using further characterization with $A p a \mathrm{I}$ and XhoI enzymes of 128 YE 4/O:3 strains [29]. However, both the time required and the costs of PFGE rise considerably when several restriction enzymes are used. The amount of working time needed for the PFGE protocol with one enzyme is two to three days, MLVA using fragment analysis can be done in one day.

In December 2003, authorities from the city of Kotka, Finland reported an outbreak of gastroenteritis. Investigations revealed that it was caused by $Y$. enterocolitica 4/O:3 [30]. Approximately 30 people fell ill; 12 patients had culture-confirmed, multiresistant YE 4/O:3 infection. Three of them had appendectomies before the disease was recognized as yersiniosis. Most of the patients had abdominal pain (94\%), fever (78\%), and diarrhea (72\%). Most of the patients had eaten in the same cafeteria in the Port of Kotka between November 25 and December 15, 2003. However, the contaminated food source was not discovered, as the food served in that cafeteria was no longer available for microbiological investigations. In addition, a cohort study among cafeteria users did not show a significant association between any food and illness. During a microbiological sampling of the cafeteria's kitchen a month later, in January 2004, hygienists noticed some shortcomings in food handling and hygiene practices that increased the possibility of cross-contamination in the cafeteria. While no YE 4/O:3 strains were found in the specimens collected from the cafeteria, YE biotype $1 \mathrm{~A}$ strains were isolated from iceberg lettuce imported from Spain and from domestic carrots. Unfortunately, the antimicrobial susceptibilities of these strains are not known. At the time of the outbreak in Kotka, there were around 20 confirmed YE 4/O:3 cases in other locations in Finland, mainly in the Turku area. The cases were suspected to be linked with the larger outbreak, but no epidemiological evidence for this was found.

MLVA played a key role in confirming that the cases which occurred in the city of Kotka in 2003 belonged to a single outbreak: 12 isolates representing the Kotka outbreak were clonal by MLVA, and differed distinctly from those of epidemiologically unrelated strains that shared the same PFGE pulsotype. Another suspicion of outbreak was refuted by MLVA: six 1-year-old children had been infected in 2006 by YE 4/O:3 strains that shared the same PFGE pulsotype (5NotI_ye a). Interviews, however, revealed no epidemiological connection between the cases. All of these strains which shared the same PFGE pulsotype were found to be of different types in MLVA. We also detected some evidence that the MLVA method can be as useful with YE 2/O:9 outbreaks as it was with YE 4/O:3. In a household outbreak in 2009, a mother and two children had YE 2/O:9 strains found to be identical in MLVA (data not shown here). MLVA also identified identical YE 2/O:9 strains in a school/day care center outbreak that occurred in Finland in 2010 (data not shown here).

Support was obtained for genetic stability among sporadic cases, since two MLVA-typed strains were isolated twice from the same patient at intervals of 7 or 19 days. In both cases, the MLVA and PFGE types remained identical. Similar observations of the stability of the $Y$. enterocolitica MLVA markers' loci in vivo had also been reported earlier [14]. Genetic events will eventually alter the MLVA patterns, but the rate of alteration is not known. However, previous studies confirmed that the MLVA type remained the same after as many as 20 serial passages of colony plating [14].

Our previous case-control study revealed that travel abroad was a risk factor for $Y$. enterocolitica infection in Finland [31]. In the present study, we found a statistically significant association between the antimicrobial multiresistance of YE strains and travel. The results indicate that a considerable number of multiresistant $Y$. enterocolitica infections are actually imported, and that domestic $Y$. enterocolitica strains in Finland are rather susceptible to antimicrobials. For instance, all of the nalidixic acid-resistant strains were isolated from patients who had been infected while on vacation in Spain or Brazil, countries where multiresistant $Y$. enterocolitica strains have been described previously $[16,25,26]$. The multiresistant strains belonged to certain PFGE pulsotypes, which were not found among susceptible strains. This is perhaps due to the DNA of the resistance plasmid. The MLVA types were so varied that no hint of the origin of the strains could be obtained on that basis.

In the outbreak that occurred in Kotka, the patients had not been abroad before falling ill. However, the antimicrobial multiresistance of the outbreak strain nevertheless suggests that the strain originated from abroad. Spanish iceberg lettuce, at least, had been used in the cafeteria. In 2005 Salmonella enterica serotype Typhimurium, with a resistance profile identical to that detected now for the $Y$. enterocolitica outbreak strain, was isolated in an outbreak situation in Finland and traced to iceberg lettuce imported from Spain [32].

The resistance of $Y$. enterocolitica to NAL is based on point mutations in the fluoroquinolone resistancedetermining regions of $\operatorname{gyr} A[26,33]$. In our study, the strains resistant to NAL had amino acid changes stemming from point mutations in the gyrA gene: i.e., either Ser83Arg, Asp87Tyr, or Asp87Asn. Two of these mutations are identical to those reported previously for fluoroquinolone-resistant $Y$. enterocolitica strains [33]. 
Conjugation experiments confirmed that in $Y$. enterocolitica, the antibiotic resistance to CHL, STR, and SUL, at least, is encoded on a large conjugative plasmid and can easily be transferred to a susceptible $Y$. enterocolitica strain. Conjugative plasmids that carry antibiotic resistance genes have been isolated from a variety of clinical strains, but reports of this for $Y$. enterocolitica are rare. Hundreds of different antibiotic resistance cassettes have been identified as residing on mobile resistance integrons [34]; owing to the cassette nature of the resistance genes, they can easily change the resistance repertoire. In fact, one of the outbreak strains in our study had altered antimicrobial resistance and lacked resistance to TET. A study on the persistence of TET-resistant E. coli in colonic microbiota observed that three out of 13 strains lost TET resistance during intestinal colonization [35].

\section{Conclusions}

MLVA was less labor-intensive than PFGE and the results were easier to analyze, especially because they were independent of subjective interpretation. PFGE can still be useful for surveillance of the sources and transmission routes of sporadic $Y$. enterocolitica strains in future. However, for outbreak investigations, MLVA offers a powerful tool for the discrimination of $Y$. enterocolitica strains. More sporadic and outbreak $Y$. enterocolitica strains should be subjected to MLVA typing in order to determine whether this method could be considered for use as a new gold standard for outbreak investigations of $Y$. enterocolitica. This study revealed that multiresistant $Y$. enterocolitica strains do appear in Finland, but that the multiresistance was mainly associated with travel. All three nalidixic acid resistant strains were associated with travel to Spain or Brazil. Interestingly, all outbreak strains studied here were also multiresistant. Thus, traditional susceptibility testing provides additional information useful for genetic typing methods in epidemiological investigations.

\section{Methods}

\section{Bacterial strains}

Sporadic $Y$. enterocolitica strains $(\mathrm{n}=82)$ of bio/serotype $4 / \mathrm{O}: 3(\mathrm{n}=75), 3 / \mathrm{O}: 3(\mathrm{n}=2), 2 / \mathrm{O}: 9(\mathrm{n}=5)$ isolated in 2006 from fecal samples of 80 Finnish patients in ten regional clinical microbiology laboratories were used in the study. The patients' mean age was 34 years (range $0.6-80$ ); $55 \%$ of them were men. Isolation and identification of the strains were described previously [36]. In addition, 22 clinical $Y$. enterocolitica strains isolated between December 2003 and January 2004, and suspected of being associated with a $Y$. enterocolitica outbreak in Kotka, were studied.

\section{MLVA}

For MLVA, we had three additional reference strains: NCTC 1176 (4/O:3); NCTC 11174 (2/O:9); and NCTC 10563 (3/O:5,27). DNA was extracted from the strains using the Jet Flex Extraction Kit (Genomed; Löhne, Germany) according to the instructions provided by the manufacturer and eluated in $100 \mu \mathrm{L}$ TE-buffer. In the MLVA analysis, six known VNTR loci of the strains were amplified in two multiplex PCRs. Previously described primers [14] were labeled with ABI PRISM ${ }^{\circledR}$ fluorescent dyes, PET, NED, 6-FAM, or VIC (Applied Biosystems, Foster City, CA). Primers were used in two separate multiplex PCRs with the VNTR loci of V2A (PET), V4 (NED), and V6 (6-FAM), as well as V5 (NED), V7 (VIC), and V9 (PET). Multiplex PCRs were performed with QIAGEN Multiplex PCR kit (Qiagen, Hilden, Germany) according to the manufacturer's instructions in a total volume of 25 $\mu \mathrm{l}$. The primer concentrations were $0.2 \mu \mathrm{M}$ (V2A), 0.16 $\mu \mathrm{M}$ (V4), and $0.2 \mu \mathrm{M}$ (V6) in the first PCR and $0.2 \mu \mathrm{M}$ (V5), $0.2 \mu \mathrm{M}$ (V7), $0.12 \mu \mathrm{M}$ (V9) in the second PCR. The template DNA concentration was approx. $10 \mathrm{ng}$. Touchdown PCR was performed with $15 \mathrm{~min}$ initial denaturation at $95^{\circ} \mathrm{C}$, followed by 9 cycles $30 \mathrm{~s}$ denaturation at $95^{\circ} \mathrm{C}$, $30 \mathrm{~s}$ annealing at $63^{\circ} \mathrm{C}-55^{\circ} \mathrm{C}$ (decreasing by $1^{\circ} \mathrm{C}$ with every cycle), and elongation at $72^{\circ} \mathrm{C}$ with an additional 25 cycles with annealing $30 \mathrm{~s}$ at $58^{\circ} \mathrm{C}$.

The two PCR products of each strain were mixed, diluted to 1/500 in sterile water, and run in capillary electrophoresis with an ABI 3730xl DNA Analyzer (Applied Biosystems, Foster City, CA) using G5 (DS-33) fragment analysis chemistry according to the manufacturer's instructions. The GeneScan ${ }^{\mathrm{TM}} 600$ LIZ $^{\circledR}$ (Applied Biosystems) was used as an internal size standard and the data were analyzed using GeneMapper v4.0 software (Applied Biosystems). MLVA types were named as a string of six numbers showing the actual number of repeat units in each of the six loci.

\section{DNA sequencing}

The selected repeats (Table 1) were sequenced in both directions with MLVA primers [14]. The gyrA gene PCR was performed for 77 sporadic $Y$. enterocolitica strains of bio/serotypes 4/O:3 and 3/O:3 with primers gyrAY1 (5'-CGC GTA CTG TTT GCG ATG AA-3') and gyrAY2 (5'-CGG AGT CAC CAT CGA CGG AA-3') as earlier described (35) (GenBank/EMBL/DDBJ accession numbers FN821873-FN821949). Sequencing was done in both directions with a Big Dye Terminator v1.1 Cycle Sequencing Kit (Applied Biosystems) with an ABI 3730xl DNA Analyzer (Applied Biosystems).

\section{PGFE}

PFGE was performed using the previously described protocol for Salmonella $[7,37]$ with modifications: Strains 
were cultured overnight at $30^{\circ} \mathrm{C}$ on R1-agar and suspended in CBS-buffer (100 mM Tris:100 MM EDTA, pH 8.0) to a final turbidity of $0.38-0.39$ at $\mathrm{A}_{480}$. Lysozyme (Roche Diagnostics GmbH, Mannheim, Germany) was added to the $400 \mu \mathrm{l}$ bacterial suspensions to reach a final concentration of $1 \mathrm{mg} / \mathrm{ml}$. The tubes were mixed and incubated for $15 \mathrm{~min}$ at $37^{\circ} \mathrm{C}$ and then heated to $50^{\circ} \mathrm{C}$, after which $400 \mu \mathrm{l}$ of $1 \%$ agarose (SeaKem Gold Agarose, Cambrex Bio Science Rockland, Inc, USA) and proteinase $\mathrm{K}$ (at a final concentration of $0.24 \mathrm{mg} / \mathrm{ml}$, Roche Diagnostics $\mathrm{GmbH}$, Mannheim, Germany) were added. The tube contents were cast into plugs, which were transferred into $3 \mathrm{ml}$ of lysis buffer (50 mM Tris:50 mM EDTA, pH8.0 + $1 \%$ Sarcosyl) containing $1 \mathrm{mg} / \mathrm{ml}$ of proteinase $\mathrm{K}$. The plugs were incubated at $54^{\circ} \mathrm{C}$ for $2 \mathrm{~h}$ and rinsed three times in sterile water and three times in TE buffer at $50^{\circ} \mathrm{C}$. The plugs were then stored in $1 \times \mathrm{TE}$ buffer at $4^{\circ} \mathrm{C}$. The released genomic DNA in the plugs was digested overnight at $37^{\circ} \mathrm{C}$ with $8 \mathrm{U}$ of the restriction enzyme Not $\mathrm{I}$ (New England Biolabs, Ipswich, MA, USA). Electrophoresis was carried out in a $1 \%$ agarose gel in $0.5 \times$ TBE buffer at $14^{\circ} \mathrm{C}$ with a switching time of 1 to $18 \mathrm{~s}$ for $40 \mathrm{~h}$ at $14^{\circ} \mathrm{C}$ with CHEF Mapper system (Bio-Rad Laboratories, Richmond, California). DNA of the Salmonella enterica serotype Braenderup strain H9812, digested with XbaI (Roche GmbH, Mannheim, Germany), was used as a size marker. The PFGE types were analyzed with Bionumerics v. 5.10 software (Applied Maths, Sint-Martens-Latem, Belgium). DNA bands smaller than $54.7 \mathrm{~kb}$ were excluded from the analysis.

\section{Discriminatory index of PFGE and MLVA}

Simpson's Index of diversity was used to calculate the discriminatory index (DI) of PFGE and MLVA [38]. In addition, the DIs of each MLVA locus was calculated.

\section{Susceptibility testing}

The antimicrobial susceptibility of the $Y$. enterocolitica isolates was determined using a set of 12 antimicrobials: ampicillin (AMP); chloramphenicol (CHL); streptomycin (STR); gentamicin (GEN); sulfonamide (SUL); tetracycline (TET); trimethoprim (TMP); ciprofloxacin (CIP); nalidixic acid (NAL); cefotaxime (CEF); mecillinam (MEC); and imipenem (IMI). The susceptibility tests were done using the agar diffusion technique on Mueller-Hinton agar according to the CLSI guidelines [39]. A strain resistant to at least four antimicrobials was called multiresistant. The minimal inhibitory concentration (MIC) for ciprofloxacin (CIP) was determined by the E-test (AB Biodisk, Solna, Sweden) for the isolates resistant to nalidixic acid, following the recommended MIC breakpoints $S \leq 1 \mathrm{mg} / \mathrm{L}$ and $\mathrm{R} \geq 4 \mathrm{mg} / \mathrm{L}$ [39]. MIC $0.125-1.0 \mathrm{mg} / \mathrm{L}$ was considered to indicate reduced susceptibility to ciprofloxacin [40].

\section{Conjugation experiments}

In conjugation experiments, the multiresistant (AMP, CHL, STR, SUL, NAL) strain YE 4/O:3 FE81008 was used as a donor strain and the kanamycin (KAN) resistant strain YeO3-U [41] as a recipient strain. Briefly, the donor strain and the recipient strain were grown overnight at room temperature shaking in $5 \mathrm{ml}$ of Luria broth (LB). The cultures were refreshed by diluting them 1:10 in LB and grown for 2-3 $\mathrm{h}$ to get them into the exponential phase. The donor strain was grown in static culture. The bacteria were then pelleted by centrifugation and resuspended in $1 \mathrm{ml}$ of PBS. After the OD600 were determined, the suspensions were mixed 1:1 and small droplets of the mixture were pipetted onto a Luria-agar plate and incubated overnight at room temperature. Only the donor or the recipient bacteria was pipetted onto the control plates. The plates were incubated overnight after which the bacteria were collected from the plates into ca. $1 \mathrm{ml}$ of PBS. Several dilutions were spread on selective plates containing CHL, KAN, or both CHL and KAN. The conjugation frequency was calculated on the basis of the proportion of CHL KAN double-resistant colonies among the CHL-resistant colonies. The resistance of the CHL KAN double-resistant colonies to the other antimicrobials was tested as described above. Plasmid isolation from $100 \mathrm{ml}$ cultures of the strains was performed using the E.Z.N.A plasmid midiprep kit (Omega Bio-Tek Inc., Norcross, GA, USA) according to the protocol provided by the manufacturer, and the plasmids were detected by running in a $1 \% \mathrm{w} / \mathrm{v}$ agarose gel.

\section{Travel information and statistical method}

Data on the patients' travel abroad were collected from the National Infectious Disease Register and from the notes of the laboratories sending the Yersinia strains for further typing. The association between travel and multiresistance was analyzed by using the chi-square method with the EpiInfo ${ }^{\mathrm{TM}}$ version 3.4.3. A p-value below 0.05 was considered to indicate statistical significance.

The study was approved by the Ethics Committee of National Institute for Health and Welfare, THL. For this study informed consents were not required as only the isolated bacterial strains of the fecal samples were studied and not the individuals themselves.

\section{Acknowledgements}

We wish to acknowledge the excellent technical assistance of Tarja Heiskanen, Kaisa Jalkanen, and Heini Flinck. Susanna Lukinmaa is acknowledged for advising with PFGE and Taru Kauko with MLVA. Elisa Huovinen and Sari Jaakola are acknowledged for collecting of the travel information. This work was supported by a grant (4850/501/2004) from the Finnish Ministry of Agriculture and Forestry. 


\section{Author details}

'Department of Infectious Disease Surveillance and Control, Bacteriology Unit, National Institute for Health and Welfare (THL), Helsinki, Fl-00271, Finland. ${ }^{2}$ Department of Infectious Disease Surveillance and Control, Epidemiological Surveillance and Response Unit, National Institute for Health and Welfare (THL), Helsinki, Fl-00271, Finland. ${ }^{3}$ Department of Bacteriology and Immunology, Haartman Institute, University of Helsinki, FI-00014, Finland. ${ }^{4}$ Helsinki University Central Hospital Laboratory Diagnostics, Helsinki, Finland.

\section{Authors' contributions}

LMS participated in the design of the study, did or supervised the MLVA, PFGE, DNA sequencing, and antimicrobial susceptibility testing, carried out the data analysis, and drafted the manuscript. ST performed the conjugation experiment. KH participated in the design of the study and drafting of the manuscript. MK did the epidemiological investigations of the study and edited the manuscript. MS designed the conjugation experiment and participated in drafting of the manuscript. AS obtained the funding, conceived the study, and edited the manuscript. All of the authors have read and approved the final manuscript.

Received: 8 September 2010 Accepted: 25 February 2011 Published: 25 February 2011

\section{References}

1. Babic-Erceg A, Klismanic Z, Erceg M, Tandara D, Smoljanovic M: An outbreak of Yersinia enterocolitica 0:3 infections on an oil tanker. Eur J Epidemiol 2003, 18(12):1159-1161.

2. Ethelberg S, Olsen KE, Gerner-Smidt P, Molbak K: Household outbreaks among culture-confirmed cases of bacterial gastrointestinal disease. Am J Epidemiol 2004, 159(4):406-412.

3. Grahek-Ogden D, Schimmer B, Cudjoe KS, Nygård K, Kapperud G: Outbreak of Yersinia enterocolitica serogroup 0:9 infection and processed pork, Norway. Emerg Infect Dis 2007, 13:754-756.

4. Jones TF: From pig to pacifier: chitterling-associated yersiniosis outbreak among black infants. Emerg Infect Dis 2003, 9(8):1007-1009.

5. Shorter NA, Thompson MD, Mooney DP, Modlin JF: Surgical aspects of an outbreak of Yersinia enterocolitis. Pediatr Surg Int 1998, 13(1):2-5.

6. Bottone EJ: Yersinia enterocolitica: the charisma continues. Clin Microbiol Rev 1997, 10(2):257-276

7. Ribot EM, Fair MA, Gautom R, Cameron DN, Hunter SB, Swaminathan B, Barrett TJ: Standardization of pulsed-field gel electrophoresis protocols for the subtyping of Escherichia coli 0157:H7, Salmonella, and Shigella for PulseNet. Foodborne Pathog Dis 2006, 3(1):59-67.

8. Asplund K, Hakkinen M, Okkonen T, Vanhala P, Nurmi E: Effects of growthpromoting antimicrobials on inhibition of Yersinia enterocolitica $0: 3$ by porcine ileal microflora. J App/ Microbiol 1998, 85(1):164-170

9. Iteman I, Guiyoule A, Carniel E: Comparison of three molecular methods for typing and subtyping pathogenic Yersinia enterocolitica strains. J Med Microbiol 1996, 45(1):48-56

10. Najdenski H, Iteman I, Carniel E: Efficient subtyping of pathogenic Yersinia enterocolitica strains by pulsed-field gel electrophoresis. J Clin Microbiol 1994, 32(12):2913-2920.

11. Saken E, Roggenkamp A, Aleksic S, Heesemann J: Characterisation of pathogenic Yersinia enterocolitica serogroups by pulsed-field gel electrophoresis of genomic Notl restriction fragments. J Med Microbiol 1994, 41(5):329-338.

12. Fredriksson-Ahomaa M, Stolle A, Korkeala H: Molecular epidemiology of Yersinia enterocolitica infections. FEMS Immunol Med Microbiol 2006, 47(3):315-329.

13. Lindstedt BA: Multiple-locus variable number tandem repeats analysis for genetic fingerprinting of pathogenic bacteria. Electrophoresis 2005, 26(13):2567-2582

14. Gierczyński R, Golubov A, Neubauer H, Pham JN, Rakin A: Development of multiple-locus variable-number tandem-repeat analysis for Yersinia enterocolitica subsp. palearctica and its application to bioserogroup 4/O3 subtyping. J Clin Microbiol 2007, 45(8):2508-2515.

15. Cornelis G, Abraham EP: Beta-lactamases from Yersinia enterocolitica. $J$ Gen Microbiol 1975, 87(2):273-284.

16. Falcao JP, Falcao DP, Pitondo-Silva A, Malaspina AC, Brocchi M: Molecular typing and virulence markers of Yersinia enterocolitica strains from human, animal and food origins isolated between 1968 and 2000 in Brazil. J Med Microbiol 2006, 55(Pt 11):1539-1548.

17. Pham JN, Bell SM, Lanzarone JY: A study of the beta-lactamases of 100 clinical isolates of Yersinia enterocolitica. J Antimicrob Chemother 1991, 28(1):19-24

18. Pham JN, Bell SM, Martin L, Carniel E: The beta-lactamases and betalactam antibiotic susceptibility of Yersinia enterocolitica. J Antimicrob Chemother 2000, 46(6):951-957.

19. Prats G, Mirelis B, Llovet T, Munoz C, Miro E, Navarro F: Antibiotic resistance trends in enteropathogenic bacteria isolated in 1985-1987 and 1995-1998 in Barcelona. Antimicrob Agents Chemother 2000, 44(5):1140-1145.

20. Stock I, Heisig P, Wiedemann B: Expression of beta-lactamases in Yersinia enterocolitica strains of biovars 2, 4 and 5. J Med Microbiol 1999, 48(11):1023-1027.

21. Bhaduri S, Wesley I, Richards H, Draughon A, Wallace M: Clonality and antibiotic susceptibility of Yersinia enterocolitica isolated from u.s. market weight hogs. Foodborne Pathog Dis 2009, 6(3):351-356.

22. Bucher M, Meyer C, Grotzbach B, Wacheck S, Stolle A, FredrikssonAhomaa M: Epidemiological data on pathogenic Yersinia enterocolitica in Southern Germany during 2000-2006. Foodborne Pathog Dis 2008 5(3):273-280

23. Mayrhofer S, Paulsen P, Smulders FJ, Hilbert F: Antimicrobial resistance profile of five major food-borne pathogens isolated from beef, pork and poultry. Int J Food Microbiol 2004, 97(1):23-29.

24. Baumgartner A, Kuffer M, Suter D, Jemmi T, Rohner P: Antimicrobial resistance of Yersinia enterocolitica strains from human patients, pigs and retail pork in Switzerland. Int J Food Microbiol 2007, 115(1):110-114.

25. Capilla S, Goni P, Rubio MC, Castillo J, Millan L, Cerda P, Sahagun J, Pitart C, Beltran A, Gomez-Lus R: Epidemiological study of resistance to nalidixic acid and other antibiotics in clinical Yersinia enterocolitica 0:3 isolates. $J$ Clin Microbiol 2003, 41(10):4876-4878.

26. Sanchez-Cespedes J, Navia MM, Martinez R, Orden B, Millan R, Ruiz J, Vila J: Clonal dissemination of Yersinia enterocolitica strains with various susceptibilities to nalidixic acid. J Clin Microbiol 2003, 41(4):1769-1771.

27. Kontiainen S, Sivonen A, Renkonen OV: Increased yields of pathogenic Yersinia enterocolitica strains by cold enrichment. Scand J Infect Dis 1994, 26:685-691.

28. Gulati P, Varshney RK, Virdi JS: Multilocus variable number tandem repeat analysis as a tool to discern genetic relationships among strains of Yersinia enterocolitica biovar 1A. J Appl Microbiol 2009

29. Fredriksson-Ahomaa M, Autio T, Korkeala H: Efficient subtyping of Yersinia enterocolitica bioserotype 4/0:3 with pulsed-field gel electrophoresis. Lett Appl Microbiol 1999, 29(5):308-312.

30. Anonymous: Infectious diseases in Finland 2003. Publications of National Public Health Institute Series B 2004, 9/2004:41.

31. Huovinen E, Sihvonen L, Virtanen M, Haukka K, Siitonen A, Kuusi M: Symptoms and sources of Yersinia enterocolitica -infection: a casecontrol study. BMC Infectious Diseases 2010, 10(1):122-131.

32. Anonymous: Infectious Diseases in Finland 2005. Publications of National Public Health Institute Series B 2006, 17/2006.

33. Capilla S, Ruiz J, Goni P, Castillo J, Rubio MC, Jimenez de Anta MT, GomezLus R, Vila J: Characterization of the molecular mechanisms of quinolone resistance in Yersinia enterocolitica 0:3 clinical isolates. J Antimicrob Chemother 2004, 53(6):1068-1071.

34. Partridge SR, Tsafnat G, Coiera E, Iredell JR: Gene cassettes and cassette arrays in mobile resistance integrons. FEMS Microbiol Rev 2009, 33(4):757-784.

35. Karami N, Nowrouzian F, Adlerberth I, Wold AE: Tetracycline resistance in Escherichia coli and persistence in the infantile colonic microbiota. Antimicrob Agents Chemother 2006, 50(1):156-161.

36. Sihvonen LM, Haukka K, Kuusi M, Virtanen MJ, Siitonen A, YE Study Group: Yersinia enterocolitica and $Y$. enterocolitica-like species in clinical stool specimens of humans: identification and prevalence of bio/serotypes in Finland. Eur J Clin Microbiol Infect Dis 2009, 28(7):757-765.

37. Lukinmaa S, Nakari UM, Liimatainen A, Siitonen A: Genomic diversity within phage types of Salmonella enterica ssp. enterica serotypes Enteritidis and Typhimurium. Foodborne Pathog Dis 2006, 3(1):97-105.

38. Hunter PR, Gaston MA: Numerical index of the discriminatory ability of typing systems: an application of Simpson's index of diversity. J Clin Microbiol 1988, 26(11):2465-2466 
39. CLSI: Performance standards for antimicrobial susceptibility testing: M100-S16. Clinical and Laboratory Standards Institute; 2006.

40. Cheasty T, Day M, Threlfall E: Increasing incidence of resistance to nalidixic acid in shigellas from humans in England and Wales: implications for therapy. Clinical Microbiology and Infection 2004, 10:1033-1035

41. Gripenberg-Lerche C, Zhang L, Ahtonen P, Toivanen P, Skurnik M: Construction of urease-negative mutants of Yersinia enterocolitica serotypes 0:3 and 0:8: role of urease in virulence and arthritogenicity. Infect Immun 2000, 68(2):942-947.

doi:10.1186/1471-2180-11-42

Cite this article as: Sihvonen et al: Multilocus Variable-Number TandemRepeat Analysis, Pulsed-Field Gel Electrophoresis, and Antimicrobial Susceptibility Patterns in Discrimination of Sporadic and OutbreakRelated Strains of Yersinia enterocolitica. BMC Microbiology 2011 11:42.

\section{Submit your next manuscript to BioMed Central} and take full advantage of:

- Convenient online submission

- Thorough peer review

- No space constraints or color figure charges

- Immediate publication on acceptance

- Inclusion in PubMed, CAS, Scopus and Google Scholar

- Research which is freely available for redistribution

Submit your manuscript at www.biomedcentral.com/submit 